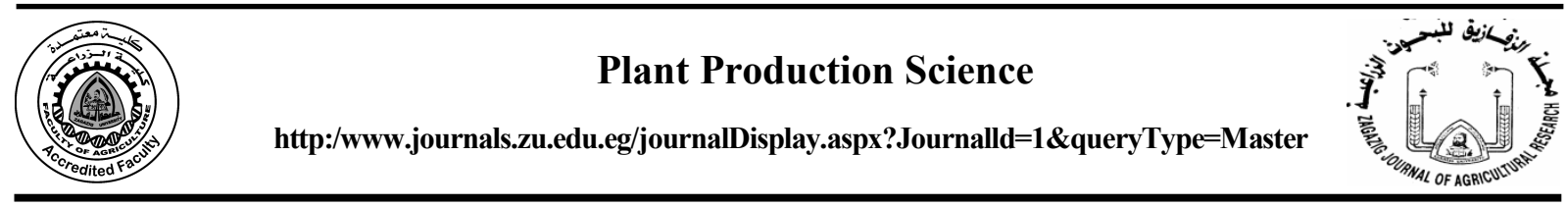

\title{
EXTENDING STORAGE PERIOD AND SHELF LIFE AND MAINTAINING QUALITY OF DILL USING MICROPERFORATED POLYPROPYLENE PACKAGES
}

\author{
Samir K. El-Seifi ${ }^{1}$, Rawia E.I. El-Bassiouny ${ }^{2}$, I.N. Nasef ${ }^{*}{ }^{*}$ and Tasnime H. Hamada ${ }^{1}$ \\ 1. Hort. Dept., Fac. Agric., Suez Canal Univ., Ismailia, Egypt \\ 2. Veg. Handling Res. Dept., Agric. Res. Cent., Giza, Egypt
}

Received: 06/01/2019 ; Accepted: 02/04/2019

\begin{abstract}
This investigation was conducted during 2016 and 2017 seasons, at the Laboratory of Horticulture Department, Faculty of Agriculture, Suez Canal University, Ismailia, Egypt, to study the effect of five types of polypropylene packages (non-perforated, perforated with 8, 16, 24 and 32 microholes) on storability and quality of dill during cold storage for $7,14,21$ and 28 days at $0 \pm 1{ }^{\circ} \mathrm{C}$, in addition to 2 and 4 days at $10 \pm 1{ }^{\circ} \mathrm{C}$ as shelf life. Weight loss, appearance, off-odor, taste, total chlorophyll, ascorbic acid, soluble solids content (SSC), total sugars content and total phenolic were measured. The results indicated that the microperforated polypropylene package with 24 microholes had the best taste and appearance, and the highest total chlorophyll, ascorbic acid, sugars and phenolic and lowest off-odor during the cold storage period at $0 \pm 1{ }^{\circ} \mathrm{C}$ for 28 days and shelf life for 4 days at 10 $\pm 1^{\circ} \mathrm{C}$ in comparison with the other packages. At the end of cold storage and period of shelf life, the microperforated polypropylene package with 24 microholes maintained the quality of dill.
\end{abstract}

Key words: Dill, microperforated polypropylene, quality, shelf life, cold storage, ascorbic acid, sugars.

\section{INTRODUCTION}

Dill (Anethum graveolens L.) is an aromatic herb. It is used for seasoning and flavoring of various foods such as sauces, salads, sea foods, soups and pickled vegetables. Leafy vegetables loss is due to the high metabolism rate which is hastened following harvest. Rapid deterioration of leafy vegetables quality via senescence is a serious problem (Koukounaras et al., 2006). Ethylene accelerates degradation of chlorophyll resulting in yellowing the leafy vegetables such as parsley (Lers et al., 1998), coriander (Jiang et al., 2002). Packaging is essential for food protection and preservation and has assumed a multi-functional role by serving as value addition symbol, quality assurance and quantity/ number, ultimately a tool for marketing food products and conveyor of convenience. The packaging importance has gained ground, thanks to the growing awareness and willingness of consumer to pay for hygienic and value produce. Stringent export market needs and increasing exports have influenced the packaging trend. Growing environmental concerns have imposed newer characteristics for package and give an impulse to the eco-friendly packaging materials development. The goal of modified atmosphere packaging in fresh commodities is to generate an atmosphere in the package with high $\mathrm{CO}_{2}$ and low $\mathrm{O}_{2}$ concentrations (relative to air) to keep the produce quality at cold temperature (Zagory, 1998; Sanz et al., 1999). MAP can be created actively by flushing a mixture of gases inside the package or passively by the respiring commodity (Church and Parson, 1995). Modified atmosphere packaging (MAP) of fresh produced focused on matching suitable plastic films to products. Because achieving a favorable modified atmosphere inside the package depends on respiration rate of product, mass of product, surface area of film, thickness of film

\footnotetext{
* Corresponding author: Tel. : +201006438356

E-mail address: innasef@hotmail.com
} 
and gas transmission rates of film. A diversity of products needs a diversity of films. The different package sizes for the similar products often needs different packaging films. Proper packaging has eased extension of products shelf life to meet the long distance distribution demands, product quality maintenance by reducing respiration rate, fruit softening and decay, browning reactions, added produce convenience through resealable packages and portion control and enhanced marketing through brands identification and improved the produce visibility (Zagory, 1997).

The objectives of this study are to provide information on the effect of microperforated polypropylene packages on fresh dill quality during cold storage at $0^{\circ} \mathrm{C}$ for 28 days and shelf life period at $10^{\circ} \mathrm{C}$.

\section{MATERIALS AND METHODS}

\section{Plant Material and Treatments}

Dill cv 'Balady' was planted in a Commercial Farm, Ismailia, Egypt, to produce the plant material that used in postharvest experiment. Dill was harvested on January $2^{\text {nd }}, 2016$ in the first season and on January $7^{\text {th }}, 2017$ in the second season. Dill was delivered to the Laboratory of Horticulture Department, Faculty of Agriculture, Suez Canal University, Ismailia, Egypt. Dill leaves were inspected visually and defected and blemished leaves were discarded. Five types of packages were used: nonperforated polypropylene package, perforated polypropylene package with 8 microholes (4 on each side of package), perforated polypropylene package with 16 microholes ( 8 on each side of package), perforated polypropylene package with 24 microholes (12 on each side of package), perforated polypropylene package with 32 microholes (16 on each side of package). The dimensions of all used packages were $17 \times 30$ $\mathrm{cm}$ and the thickness was $40 \mu \mathrm{m}$. The diameter of microhole is $540 \mu \mathrm{m}$. One hundred gram of dill leaves (20-25 cm long) with green fully was packed in each package, then was closed by a heat sealer. All treatments were made in triplicate and stored at $0 \pm 1^{\circ} \mathrm{C}$, with $90-95 \% \mathrm{RH}$ for $7,14,21$ and 28 days. The dill which was stored at $0^{\circ} \mathrm{C}$ for $7,14,21$ and 28 days were kept for 2 and 4 days at $10 \pm 1{ }^{\circ} \mathrm{C}$, with $80-85 \%$ $\mathrm{RH}$, in order to simulate the retail sale period.

\section{Assessment and Measurements}

On each day of analysis, 9 replicates were randomly taken out of the $0^{\circ} \mathrm{C}$ storage from each package, 3 replicates were evaluated at the same day, and 6 replicates kept at $10^{\circ} \mathrm{C}$ for $2(3$ replicates) and 4 (3 replicates) days as a simulated retail sale period.

\section{Weight loss (\%)}

The weight of dill packages were measured on zero time and on the day of sampling. During the storage, the weight loss of dill was calculated by determining the changes in weight and calculated as the percentage of initial fresh weight.

\section{Appearance}

A group consists of five drilled panelists judged the appearance of dill from each package. The typical characteristics of the dill and the possibilities of impairment were demonstrated before the evaluation started. Visual quality of dill herbs were recorded on 9 to 1 scale, where $9=$ excellent (fresh appearance), $7=$ very good, $5=$ good (limited marketability), $3=$ fair (limit of usability) and $1=$ poor, inedible according to Koukounaras et al. (2008). The middle numbers were appointed where convenient. The term "storage life" indicate to the time needed for the sample to deteriorate from ranking 9 (field fresh) to 1 (poor).

\section{Taste}

The taste assessment was completed by a trained group of 5 peoples in a specially furnisher room with individual booths. The panelists were trained on the sensory features of fresh dill and were experienced in tasting fresh produce. The panel members evaluated 3 replicates for each treatment and used water for washing after tasting dill from each package. Panelists ranked dill for taste on a scale of five to one, where $5=$ fully typical, $4=$ moderately full, $3=$ moderate, $2=$ slight and $1=$ none (Elwan et al., 2015).

\section{Off odor}

Off odor was estimated on a scale of five to one, where $5=$ severe, $4=$ moderately severe, 3 = moderate, $2=$ slight and $1=$ none $($ Elwan $\boldsymbol{e t}$ al., 2015). 


\section{Total chlorophyll contents}

Total chlorophyll was extracted ( $0.5 \mathrm{~g})$ by $80 \%$ acetone and measured spectrophotometricaly according to Lichenthaler and Wellburn (1983), then calculated as $\mathrm{mg} / 100 \mathrm{~g}$.

\section{Ascorbic acid content}

Dill leaves $(10 \mathrm{~g})$ were ground in a mortar with $50 \mathrm{ml}$ of $0.9 \mathrm{M}$ oxalic acid. The mixture was transferred to volumetric flask, then made up to $100 \mathrm{ml}$ with distilled water and filtered by a paper of filter (Whatman No 1), and then ten milliliter of supernatant were titrated to pink color using $0.1 \%$ 2,6-dichlorophenolindophenol titration, and then ascorbic acid was calculated and expressed as $\mathrm{mg} / 100 \mathrm{~g}$ fresh weight according to Pearson (1970).

\section{Soluble solids content}

Soluble solids content (SSC) of dill was measured by hand rafractometer according to AOAC (1996) expressed as (\%) at $20^{\circ} \mathrm{C}$.

\section{Total sugars}

Five grams of fresh dill were homogenized for two $\mathrm{min}$ in $50 \mathrm{ml}$ of $80 \%$ ethanol, and then refluxed for thirty minutes, and was centrifuged for $30 \mathrm{~min}$ at $10000 \times \mathrm{g}$. The residue was exposed to ethanol extraction anther time. The extractions were collected and the alcohol was separated via evaporation. The aliquot was filled with distilled water up to $50 \mathrm{ml}$. The total sugars were determined with phenol-sulfuric acid reagent using spectrophotometer at $480 \mathrm{~nm}$ according to Dubois et al. (1956).

\section{Total phenolic content}

Total phenolic determination was carried out for dill according to Mazumdar and Majumder (2003) as described in the following method. Total phenolic was extracted from $5 \mathrm{~g}$ of fresh dill (each sample date) with $80 \%$ ethanol in boiling water bath and then was cooled to room temperature. After cooling, the extract was centrifuged. The supernatant was evaporated to nearly dryness in a water path, then the residue was dissolved in distilled water and made up to $100 \mathrm{ml}$ with distilled water, from this solution $1.00 \mathrm{ml}$ was taken into test tube, then $0.5 \mathrm{ml}$ of folin ciocalteu's phenol reagent was added.
After 5 minutes, $1 \mathrm{ml}$ of $15 \%$ sodium carbonate was added and then made up to $10 \mathrm{ml}$ with distilled water. The optical density of the blue color was measured through $650 \mathrm{~nm}$ wavelength in a spectrophotometer, then calculated as $\mathrm{mg} / 100 \mathrm{~g}$ fresh weight.

\section{Statistical Analysis}

Data were organized in a completely randomized block design (CRBD) with a split plot arrangement the packages were randomly circulated in the main plots and the times of storage were randomly circulated in the subplots, with three replications, in which each replicate was considered as a block. Least significance difference (LSD) test was used to compare means at the $5 \%$ significance level. Experimental data were statistically evaluated by COStat version 6.303 1998-2004 CoHort software 798 lighthouse Ave PMP 320, Monterey, CA, 93940, USA. Analysis of variance (ANOVA) was achieved to compare results.

\section{RESULTS}

\section{Weight Loss (\%)}

The influences of packages, periods of storage and their interaction on dill weight loss (\%) during 28 days at $0^{\circ} \mathrm{C}$ of cold storage plus 2 and 4 days of shelf life at $10{ }^{\circ} \mathrm{C}$ during the two seasons are shown in Fig. 1. The results showed that the perforated polypropylene package with 32 microholes (32 hr.) had the highest weight loss (\%) while the perforated polypropylene package with 8 microholes ( $8 \mathrm{hr}$.) had the lowest. The weight loss (\%) increased with elongation of cold storage and period of shelf life. Concerning the interaction effects on weight loss (\%), no weight loss was recorded in the non-perforated polypropylene package and the perforated polypropylene package with 8 microholes during 7 days at $0^{\circ} \mathrm{C}$. At the end of storage, the perforated polypropylene package with 32 microholes (32 hr.) had the highest weight loss. However, no significant differences were noticed between the other packages (nonperforated polypropylene package the perforated polypropylene packages with 8,16 and 24 microholes). 


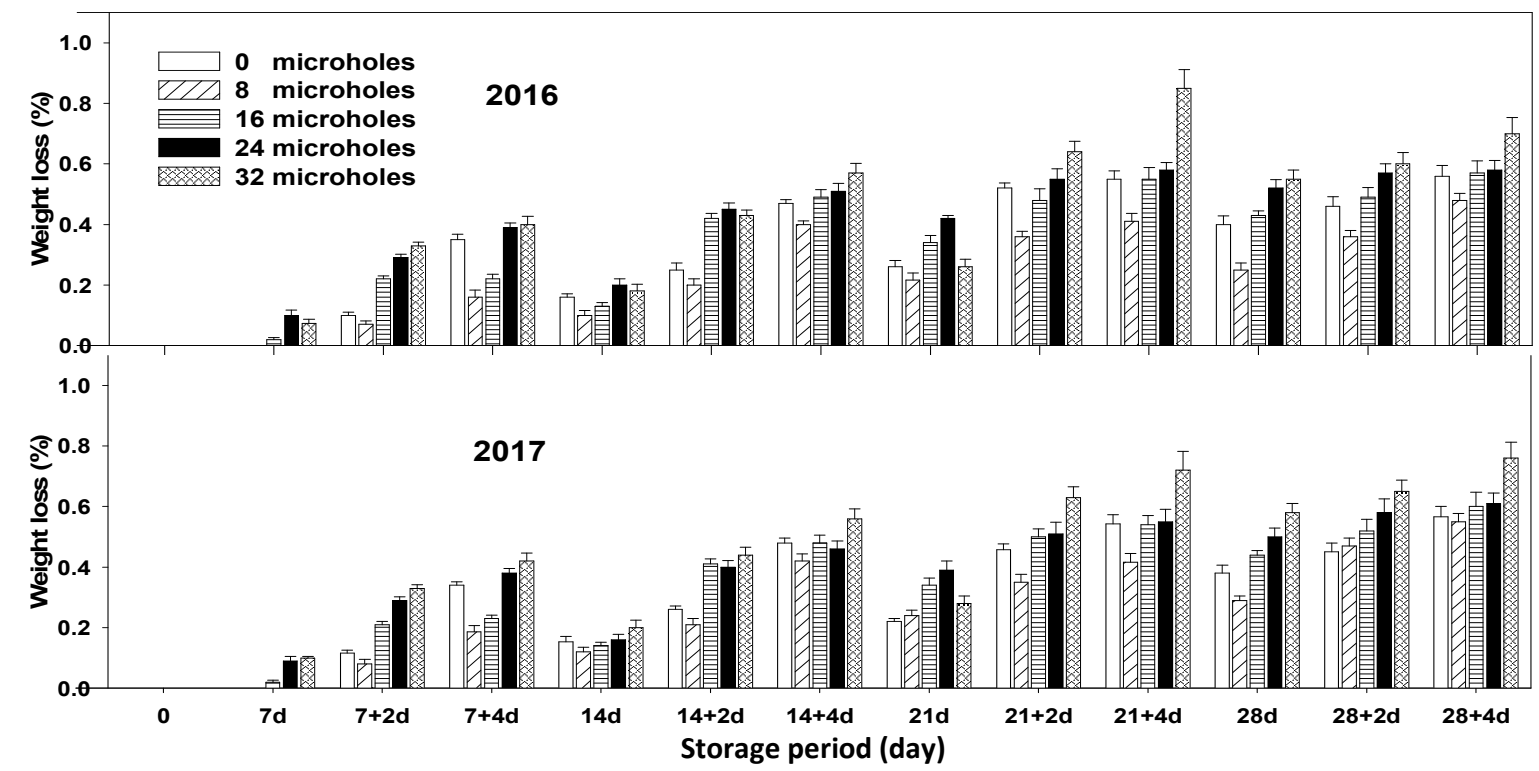

Fig. 1. Effect of microperforated polypropylene packages on weight loss (\%) of dill during 2016 and 2017 seasons

\section{Appearance}

The results in Table 1 reveal that appearance of dill was significantly affected by packages, storage periods and their interaction during the two seasons (2016-2017) $(\mathrm{p}<0.001)$. The perforated polypropylene package with 24 microholes $(24$ hr.) had the highest value of appearance compared with the other packages. The appearance decreased by extension of storage period and this decreasing was marked when the packages were transferred to the retail sale at $10{ }^{\circ} \mathrm{C}$. Regarding the interaction of packages and storage periods, the appearance decreased sharply in non-perforated polypropylene package after 14 days at $0^{\circ} \mathrm{C}$ plus 2 days at $10^{\circ} \mathrm{C}$, then the samples lost its appearance after 21 days at $0^{\circ} \mathrm{C}$ and 4 days at $10^{\circ} \mathrm{C}$. The perforated polypropylene packages with 16 and 24 microholes maintained the appearance of dill at 9 scores (fresh and excellent appearance) after 21 days at $0^{\circ} \mathrm{C}$. However, the perforated polypropylene packages with 24 microholes had 5 scores which mean good appearance after 28 days at $0^{\circ} \mathrm{C}$ plus 2 days at $10^{\circ} \mathrm{C}$ compared with the other packages.

\section{Off Odor}

Results in Table 2 show the effect of packages, storage periods and their interaction on off odor during the two seasons. Nonperforated polypropylene package had the highest off odor while the perforated polypropylene package with 24 microholes had the lowest value. The off odor increased by extension of storage period in both seasons. In regard to the interaction, off odor was detected in non-perforated polypropylene packages after 7 days at $0^{\circ} \mathrm{C}$ and 2 days at $10^{\circ} \mathrm{C}$ then reached to 5 scores (severe) after period of cold storage for 21 days at $0^{\circ} \mathrm{C}$. However, off odor was not detected in the perforated polypropylene packages $(8,16,24$ and 32 microholes) until 21 days at $0{ }^{\circ} \mathrm{C}$ plus 2 days at $10^{\circ} \mathrm{C}$. At the end of storage period, perforated polypropylene package with 24 microholes had 2 scores (slight) in comparison with the other packages.

\section{Taste}

Results in Table 3 show the effect of packages, storage periods and their interaction on dill taste during two seasons. The perforated polypropylene package had the highest taste, while the non-perforated polypropylene package had the lowest taste in both seasons. The dill taste decreased gradually during storage periods. Concerning the interaction, the taste reduced severely in non-perforated polypropylene package and then the samples lost its taste after 21 days at $0^{\circ} \mathrm{C}$. However, the dill packed in perforated polypropylene packages with 16 and 24 microholes kept its taste (fully typical) up to 21 days of cold storage at $0^{\circ} \mathrm{C}$. 
Zagazig J. Agric. Res., Vol. 46 No. (2) 2019

Table 1. Effect of microperforated polypropylene packages on appearance of dill during 2016 and 2017 seasons

\begin{tabular}{|c|c|c|c|c|c|c|}
\hline \multirow[t]{2}{*}{ Treatment } & \multicolumn{5}{|c|}{ Package } & \multirow[t]{2}{*}{ Mean } \\
\hline & Non-perforated & 8 holes & 16 holes & 24 holes & 32 holes & \\
\hline & \multicolumn{5}{|c|}{2016 season } & \\
\hline 0 time & 9.00 & 9.00 & 9.00 & 9.00 & 9.00 & \\
\hline $7 d^{1}$ & $9.00 \mathrm{a}$ & $9.00 \mathrm{a}$ & $9.00 \mathrm{a}$ & $9.00 \mathrm{a}$ & $9.00 \mathrm{a}$ & $9.00 \mathrm{~A}$ \\
\hline $7+2 d^{2}$ & $7.00 \mathrm{~cd}$ & $9.00 \mathrm{a}$ & $9.00 \mathrm{a}$ & $9.00 \mathrm{a}$ & $9.00 \mathrm{a}$ & $8.60 \mathrm{~B}$ \\
\hline $7+4 d^{2}$ & $7.00 \mathrm{~cd}$ & $9.00 \mathrm{a}$ & $9.00 \mathrm{a}$ & $9.00 \mathrm{a}$ & $9.00 \mathrm{a}$ & $8.60 \mathrm{~B}$ \\
\hline $14 d^{1}$ & $7.00 \mathrm{~cd}$ & $9.00 \mathrm{a}$ & $9.00 \mathrm{a}$ & $9.00 \mathrm{a}$ & $9.00 \mathrm{a}$ & $8.60 \mathrm{~B}$ \\
\hline $14+2 d^{2}$ & $4.33 \mathrm{gh}$ & $7.67 \mathrm{bc}$ & $9.00 \mathrm{a}$ & $9.00 \mathrm{a}$ & $7.67 b c$ & $7.53 \mathrm{C}$ \\
\hline $14+4 d^{2}$ & 3.67hi & $7.00 \mathrm{~cd}$ & $8.33 \mathrm{ab}$ & $8.33 \mathrm{ab}$ & $7.00 \mathrm{~cd}$ & $6.87 \mathrm{D}$ \\
\hline $21 d^{1}$ & $4.33 \mathrm{gh}$ & $7.00 \mathrm{~cd}$ & $9.00 \mathrm{a}$ & $9.00 \mathrm{a}$ & $8.33 \mathrm{ab}$ & $7.53 \mathrm{C}$ \\
\hline $21+2 d^{2}$ & $3.00 \mathrm{ij}$ & $6.33 \mathrm{de}$ & $7.00 \mathrm{~cd}$ & $7.00 \mathrm{~cd}$ & $6.33 \mathrm{de}$ & $5.93 \mathrm{E}$ \\
\hline $21+4 d^{2}$ & $2.33 \mathrm{j}$ & $4.33 \mathrm{gh}$ & $5.00 \mathrm{fg}$ & $5.00 \mathrm{fg}$ & $5.00 \mathrm{fg}$ & $4.33 \mathrm{~F}$ \\
\hline $28 d^{1}$ & $1.00 \mathrm{k}$ & $4.33 \mathrm{gh}$ & $5.00 \mathrm{fg}$ & $5.67 \mathrm{ef}$ & $4.33 \mathrm{gh}$ & $4.07 \mathrm{~F}$ \\
\hline $28+2 d^{2}$ & $1.00 \mathrm{k}$ & $3.00 \mathrm{ij}$ & $3.00 \mathrm{ij}$ & $5.00 \mathrm{fg}$ & $3.00 \mathrm{ij}$ & $3.00 \mathrm{G}$ \\
\hline $28+4 d^{2}$ & $1.00 \mathrm{k}$ & $2.33 \mathrm{j}$ & $3.00 \mathrm{ij}$ & $3.00 \mathrm{ij}$ & $1.00 \mathrm{k}$ & $2.07 \mathrm{H}$ \\
\hline \multirow[t]{2}{*}{ Mean } & $4.22 \mathrm{C}$ & $6.50 \mathrm{~B}$ & 7.11A & $7.33 \mathrm{~A}$ & $6.56 \mathrm{~B}$ & \\
\hline & \multicolumn{5}{|c|}{2017 season } & \\
\hline 0 time & 9.00 & 9.00 & 9.00 & 9.00 & 9.00 & \\
\hline $7 d^{1}$ & $9.00 \mathrm{a}$ & $9.00 \mathrm{a}$ & $9.00 \mathrm{a}$ & $9.00 \mathrm{a}$ & $9.00 \mathrm{a}$ & $9.00 \mathrm{~A}$ \\
\hline $7+2 d^{2}$ & $7.00 \mathrm{~cd}$ & $9.00 \mathrm{a}$ & $9.00 \mathrm{a}$ & $9.00 \mathrm{a}$ & $9.00 \mathrm{a}$ & 8.60AB \\
\hline $7+4 d^{2}$ & $7.00 \mathrm{~cd}$ & $9.00 \mathrm{a}$ & $9.00 \mathrm{a}$ & $9.00 \mathrm{a}$ & $9.00 \mathrm{a}$ & $8.60 \mathrm{AB}$ \\
\hline $14 d^{1}$ & $6.33 \mathrm{de}$ & $8.33 \mathrm{ab}$ & $9.00 \mathrm{a}$ & $9.00 \mathrm{a}$ & $9.00 \mathrm{a}$ & $8.33 \mathrm{~B}$ \\
\hline $14+2 d^{2}$ & $4.33 \mathrm{~g}$ & $7.67 \mathrm{bc}$ & $8.33 \mathrm{ab}$ & $9.00 \mathrm{a}$ & $7.67 \mathrm{bc}$ & $7.40 \mathrm{C}$ \\
\hline $14+4 d^{2}$ & $3.00 \mathrm{~h}$ & $7.00 \mathrm{~cd}$ & $8.33 \mathrm{ab}$ & $8.33 \mathrm{ab}$ & $7.00 \mathrm{~cd}$ & $6.73 \mathrm{D}$ \\
\hline $21 d^{1}$ & $4.33 \mathrm{~g}$ & $7.00 \mathrm{~cd}$ & $9.00 \mathrm{a}$ & $9.00 \mathrm{a}$ & $7.67 \mathrm{bc}$ & $7.40 \mathrm{C}$ \\
\hline $21+2 d^{2}$ & $3.00 \mathrm{~h}$ & $6.33 \mathrm{de}$ & $6.33 \mathrm{de}$ & $6.33 \mathrm{de}$ & $6.33 \mathrm{de}$ & $5.67 \mathrm{E}$ \\
\hline $21+4 d^{2}$ & $1.67 \mathrm{i}$ & $4.33 \mathrm{~g}$ & $5.00 \mathrm{fg}$ & $5.67 \mathrm{ef}$ & $5.00 \mathrm{fg}$ & $4.33 \mathrm{~F}$ \\
\hline $28 d^{1}$ & $1.00 \mathrm{i}$ & $4.33 \mathrm{~g}$ & $5.00 \mathrm{fg}$ & $5.67 \mathrm{ef}$ & $4.33 \mathrm{~g}$ & $4.07 \mathrm{~F}$ \\
\hline $28+2 d^{2}$ & $1.00 \mathrm{i}$ & $3.00 \mathrm{~h}$ & $3.00 \mathrm{~h}$ & $5.00 \mathrm{fg}$ & $3.00 \mathrm{~h}$ & $3.00 \mathrm{G}$ \\
\hline $28+4 d^{2}$ & $1.00 \mathrm{i}$ & $1.67 \mathrm{i}$ & $3.00 \mathrm{~h}$ & $3.00 \mathrm{~h}$ & $1.00 \mathrm{i}$ & $1.93 \mathrm{H}$ \\
\hline Mean & 4.06D & $6.39 \mathrm{C}$ & 7.00B & 7.33A & $6.50 \mathrm{C}$ & \\
\hline
\end{tabular}

The values within a column with different letters are significantly different at $\mathrm{P} \leq 0.001 \%$ according to the LSD multiple range test. 1: Cold storage period, 2 : Cold storage period + shelf life. 
Table 2. Effect of microperforated polypropylene packages on off odor of dill during 2016 and 2017 seasons

\begin{tabular}{|c|c|c|c|c|c|c|}
\hline \multirow[t]{2}{*}{ Treatment } & \multicolumn{5}{|c|}{ Package } & \multirow[t]{2}{*}{ Mean } \\
\hline & Non-perforated & 8 holes & 16 holes & 24 holes & 32 holes & \\
\hline \multicolumn{7}{|c|}{2016 season } \\
\hline 0 time & 1.00 & 1.00 & 1.00 & 1.00 & 1.00 & \\
\hline $7 d^{1}$ & $1.00 \mathrm{i}$ & $1.00 \mathrm{i}$ & $1.00 \mathrm{i}$ & $1.00 \mathrm{i}$ & $1.00 \mathrm{i}$ & $1.00 \mathrm{H}$ \\
\hline $7+2 d^{2}$ & $2.00 \mathrm{~g}$ & $1.00 \mathrm{i}$ & $1.00 \mathrm{i}$ & $1.00 \mathrm{i}$ & $1.00 \mathrm{i}$ & $1.20 \mathrm{G}$ \\
\hline $7+4 d^{2}$ & $3.00 \mathrm{~d}$ & $1.00 \mathrm{i}$ & $1.00 \mathrm{i}$ & $1.00 \mathrm{i}$ & $1.00 \mathrm{i}$ & $1.40 \mathrm{~F}$ \\
\hline $14 d^{1}$ & $2.00 \mathrm{~g}$ & $1.00 \mathrm{i}$ & $1.00 \mathrm{i}$ & $1.00 \mathrm{i}$ & $1.00 \mathrm{i}$ & $1.20 \mathrm{~g}$ \\
\hline $14+2 d^{2}$ & $3.00 \mathrm{~d}$ & $1.00 \mathrm{i}$ & $1.00 \mathrm{i}$ & $1.00 \mathrm{i}$ & $1.00 \mathrm{i}$ & $1.40 \mathrm{~F}$ \\
\hline $14+4 d^{2}$ & $4.00 \mathrm{c}$ & $1.00 \mathrm{i}$ & $1.00 \mathrm{i}$ & $1.00 \mathrm{i}$ & $1.00 \mathrm{i}$ & $1.60 \mathrm{E}$ \\
\hline $21 d^{1}$ & $3.00 \mathrm{~d}$ & $1.00 \mathrm{i}$ & $1.00 \mathrm{i}$ & $1.00 \mathrm{i}$ & $1.00 \mathrm{i}$ & $1.40 \mathrm{~F}$ \\
\hline $21+2 d^{2}$ & $4.00 \mathrm{c}$ & $2.00 \mathrm{~g}$ & $2.00 \mathrm{~g}$ & $2.00 \mathrm{~g}$ & $2.00 \mathrm{~g}$ & $2.40 \mathrm{D}$ \\
\hline $21+4 d^{2}$ & $4.33 b$ & $2.00 \mathrm{~g}$ & $2.00 \mathrm{~g}$ & $2.00 \mathrm{~g}$ & $2.00 \mathrm{~g}$ & $2.47 \mathrm{D}$ \\
\hline $28 d^{1}$ & $5.00 \mathrm{a}$ & $2.00 \mathrm{~g}$ & $2.00 \mathrm{~g}$ & $1.67 \mathrm{~h}$ & $2.33 f$ & $2.60 \mathrm{C}$ \\
\hline $28+2 d^{2}$ & $5.00 \mathrm{a}$ & $2.67 \mathrm{e}$ & $2.00 \mathrm{~g}$ & $2.00 \mathrm{~g}$ & $2.00 \mathrm{~g}$ & $2.73 \mathrm{~B}$ \\
\hline $28+4 d^{2}$ & $5.00 \mathrm{a}$ & $3.00 \mathrm{~d}$ & $2.67 \mathrm{e}$ & $2.00 \mathrm{~g}$ & $3.00 \mathrm{~d}$ & $3.13 \mathrm{~A}$ \\
\hline Mean & $3.44 \mathrm{~A}$ & $1.56 \mathrm{~B}$ & $1.47 \mathrm{C}$ & $1.39 \mathrm{D}$ & $1.53 B$ & \\
\hline \multicolumn{7}{|c|}{2017 season } \\
\hline 0 time & 1.00 & 1.00 & 1.00 & 1.00 & 1.00 & \\
\hline $7 d^{1}$ & $1.00 \mathrm{~h}$ & $1.00 \mathrm{~h}$ & $1.00 \mathrm{~h}$ & $1.00 \mathrm{~h}$ & $1.00 \mathrm{~h}$ & $1.00 \mathrm{H}$ \\
\hline $7+2 d^{2}$ & $2.00 \mathrm{f}$ & $1.00 \mathrm{~h}$ & $1.00 \mathrm{~h}$ & $1.00 \mathrm{~h}$ & $1.00 \mathrm{~h}$ & $1.20 \mathrm{G}$ \\
\hline $7+4 d^{2}$ & $3.00 \mathrm{~d}$ & $1.00 \mathrm{~h}$ & $1.00 \mathrm{~h}$ & $1.00 \mathrm{~h}$ & $1.00 \mathrm{~h}$ & $1.40 \mathrm{~F}$ \\
\hline $14 d^{1}$ & $2.00 \mathrm{f}$ & $1.00 \mathrm{~h}$ & $1.00 \mathrm{~h}$ & $1.00 \mathrm{~h}$ & $1.00 \mathrm{~h}$ & $1.20 \mathrm{G}$ \\
\hline $14+2 d^{2}$ & $3.00 \mathrm{~d}$ & $1.00 \mathrm{~h}$ & $1.00 \mathrm{~h}$ & $1.00 \mathrm{~h}$ & $1.00 \mathrm{~h}$ & $1.40 \mathrm{~F}$ \\
\hline $14+4 d^{2}$ & $4.00 \mathrm{c}$ & $1.00 \mathrm{~h}$ & $1.00 \mathrm{~h}$ & $1.00 \mathrm{~h}$ & $1.00 \mathrm{~h}$ & $1.60 \mathrm{E}$ \\
\hline $21 d^{1}$ & $3.00 \mathrm{~d}$ & $1.00 \mathrm{~h}$ & $1.00 \mathrm{~h}$ & $1.00 \mathrm{~h}$ & $1.00 \mathrm{~h}$ & $1.40 \mathrm{~F}$ \\
\hline $21+2 d^{2}$ & $4.00 \mathrm{c}$ & $2.00 \mathrm{f}$ & $2.00 \mathrm{f}$ & $1.67 \mathrm{~g}$ & $2.00 \mathrm{f}$ & $2.33 \mathrm{D}$ \\
\hline $21+4 d^{2}$ & $4.67 \mathrm{~b}$ & $2.00 \mathrm{f}$ & $2.00 \mathrm{f}$ & $2.00 \mathrm{f}$ & $2.00 \mathrm{f}$ & $2.53 \mathrm{C}$ \\
\hline $28 d^{1}$ & $5.00 \mathrm{a}$ & $2.00 \mathrm{f}$ & $2.00 \mathrm{f}$ & $1.67 \mathrm{~g}$ & $2.00 \mathrm{f}$ & $2.53 \mathrm{C}$ \\
\hline $28+2 d^{2}$ & $5.00 \mathrm{a}$ & $2.67 \mathrm{e}$ & $2.00 \mathrm{f}$ & $1.67 \mathrm{~g}$ & $2.00 \mathrm{f}$ & $2.67 \mathrm{~B}$ \\
\hline $28+4 d^{2}$ & $5.00 \mathrm{a}$ & $3.00 \mathrm{~d}$ & $2.67 \mathrm{e}$ & $2.00 \mathrm{f}$ & $3.00 \mathrm{~d}$ & $3.13 \mathrm{~A}$ \\
\hline Mean & $3.47 \mathrm{~A}$ & $1.56 \mathrm{~B}$ & $1.47 \mathrm{~B}$ & $1.33 \mathrm{C}$ & $1.50 \mathrm{~B}$ & \\
\hline
\end{tabular}

The values within a column with different letters are significantly different at $\mathrm{P} \leq 0.001 \%$ according to the LSD multiple range test. 1: Cold storage period, 2: Cold storage period + shelf life. 
Zagazig J. Agric. Res., Vol. 46 No. (2) 2019

Table 3. Effect of microperforated polypropylene packages on tast of dill during 2016 and 2017 seasons

\begin{tabular}{|c|c|c|c|c|c|c|}
\hline \multirow[t]{2}{*}{ Treatment } & \multicolumn{5}{|c|}{ Package } & \multirow[t]{2}{*}{ Mean } \\
\hline & Non-perforated & 8 holes & 16 holes & 24 holes & 32 holes & \\
\hline \multicolumn{7}{|c|}{2016 season } \\
\hline 0 time & 5.00 & 5.00 & 5.00 & 5.00 & 5.00 & \\
\hline $7 d^{1}$ & $5.00 \mathrm{a}$ & $5.00 \mathrm{a}$ & $5.00 \mathrm{a}$ & $5.00 \mathrm{a}$ & $5.00 \mathrm{a}$ & $5.00 \mathrm{~A}$ \\
\hline $7+2 d^{2}$ & $4.00 \mathrm{~b}$ & $5.00 \mathrm{a}$ & $5.00 \mathrm{a}$ & $5.00 \mathrm{a}$ & $5.00 \mathrm{a}$ & $4.80 \mathrm{~B}$ \\
\hline $7+4 d^{2}$ & $3.33 \mathrm{~cd}$ & $5.00 \mathrm{a}$ & $5.00 \mathrm{a}$ & $5.00 \mathrm{a}$ & $5.00 \mathrm{a}$ & 4.67B-D \\
\hline $14 d^{1}$ & $3.67 b c$ & $5.00 \mathrm{a}$ & $5.00 \mathrm{a}$ & $5.00 \mathrm{a}$ & $5.00 \mathrm{a}$ & $4.73 \mathrm{BC}$ \\
\hline $14+2 d^{2}$ & $3.00 \mathrm{~d}$ & $5.00 \mathrm{a}$ & $5.00 \mathrm{a}$ & $5.00 \mathrm{a}$ & $5.00 \mathrm{a}$ & $4.60 \mathrm{CD}$ \\
\hline $14+4 d^{2}$ & $2.00 \mathrm{e}$ & $5.00 \mathrm{a}$ & $5.00 \mathrm{a}$ & $5.00 \mathrm{a}$ & $4.67 \mathrm{a}$ & $4.33 \mathrm{E}$ \\
\hline $21 d^{1}$ & $3.33 \mathrm{~cd}$ & $4.67 \mathrm{a}$ & $5.00 \mathrm{a}$ & $5.00 \mathrm{a}$ & $4.67 \mathrm{a}$ & $4.53 \mathrm{D}$ \\
\hline $21+2 d^{2}$ & $3.00 \mathrm{~d}$ & $4.00 \mathrm{~b}$ & $4.00 \mathrm{~b}$ & $4.00 \mathrm{~b}$ & $4.00 \mathrm{~b}$ & $3.80 \mathrm{~F}$ \\
\hline $21+4 d^{2}$ & $2.00 \mathrm{e}$ & $4.00 \mathrm{~b}$ & $4.00 \mathrm{~b}$ & $4.00 \mathrm{~b}$ & $4.00 \mathrm{~b}$ & $3.60 \mathrm{G}$ \\
\hline $28 d^{1}$ & $1.00 \mathrm{f}$ & $4.00 \mathrm{~b}$ & $4.67 \mathrm{a}$ & $4.67 \mathrm{a}$ & $4.00 \mathrm{~b}$ & $3.67 \mathrm{FG}$ \\
\hline $28+2 d^{2}$ & $1.00 \mathrm{f}$ & $3.33 \mathrm{~cd}$ & $3.67 b c$ & $4.00 \mathrm{~b}$ & $3.00 \mathrm{~d}$ & $3.00 \mathrm{H}$ \\
\hline $28+4 d^{2}$ & $1.00 \mathrm{f}$ & $3.00 \mathrm{~d}$ & $3.00 \mathrm{~d}$ & $3.00 \mathrm{~d}$ & $1.00 \mathrm{f}$ & $2.20 \mathrm{I}$ \\
\hline Mean & 2.69D & 4.42B & $4.53 \mathrm{~A}$ & $4.56 \mathrm{~A}$ & 4.19C & \\
\hline \multicolumn{7}{|c|}{2017 season } \\
\hline 0 time & 5.00 & 5.00 & 5.00 & 5.00 & 5.00 & \\
\hline $7 d^{1}$ & $5.00 \mathrm{a}$ & $5.00 \mathrm{a}$ & $5.00 \mathrm{a}$ & $5.00 \mathrm{a}$ & $5.00 \mathrm{a}$ & $5.00 \mathrm{~A}$ \\
\hline $7+2 d^{2}$ & $4.00 \mathrm{~cd}$ & $5.00 \mathrm{a}$ & $5.00 \mathrm{a}$ & $5.00 \mathrm{a}$ & $5.00 \mathrm{a}$ & $4.80 \mathrm{AB}$ \\
\hline $7+4 d^{2}$ & $3.33 \mathrm{ef}$ & $5.00 \mathrm{a}$ & $5.00 \mathrm{a}$ & $5.00 \mathrm{a}$ & $5.00 \mathrm{a}$ & $4.67 \mathrm{BC}$ \\
\hline $14 d^{1}$ & $3.67 \mathrm{de}$ & $3.67 \mathrm{de}$ & $5.00 \mathrm{a}$ & $5.00 \mathrm{a}$ & $5.00 \mathrm{a}$ & $4.73 \mathrm{~B}$ \\
\hline $14+2 d^{2}$ & $3.00 \mathrm{fg}$ & $5.00 \mathrm{a}$ & $5.00 \mathrm{a}$ & $5.00 \mathrm{a}$ & $5.00 \mathrm{a}$ & $4.60 \mathrm{BC}$ \\
\hline $14+4 d^{2}$ & $2.00 \mathrm{~h}$ & $5.00 \mathrm{a}$ & $5.00 \mathrm{a}$ & $5.00 \mathrm{a}$ & $4.67 \mathrm{ab}$ & $4.33 \mathrm{D}$ \\
\hline $21 d^{1}$ & $3.33 \mathrm{ef}$ & $4.33 b c$ & $5.00 \mathrm{a}$ & $5.00 \mathrm{a}$ & $4.67 \mathrm{ab}$ & 4.47CD \\
\hline $21+2 d^{2}$ & $3.00 \mathrm{fg}$ & $4.00 \mathrm{~cd}$ & $4.00 \mathrm{~cd}$ & $4.00 \mathrm{~cd}$ & $4.00 \mathrm{~cd}$ & $3.80 \mathrm{E}$ \\
\hline $21+4 d^{2}$ & $1.33 \mathrm{i}$ & $3.67 \mathrm{de}$ & $3.67 \mathrm{de}$ & $4.00 \mathrm{~cd}$ & $4.00 \mathrm{~cd}$ & $3.33 \mathrm{~F}$ \\
\hline $28 d^{1}$ & $1.00 \mathrm{i}$ & $3.67 \mathrm{de}$ & $4.00 \mathrm{~cd}$ & $4.67 \mathrm{ab}$ & $3.67 \mathrm{de}$ & $3.40 \mathrm{~F}$ \\
\hline $28+2 d^{2}$ & $1.00 \mathrm{i}$ & $3.00 \mathrm{fg}$ & $3.33 \mathrm{ef}$ & $4.00 \mathrm{~cd}$ & $2.67 \mathrm{~g}$ & $2.80 \mathrm{G}$ \\
\hline $28+4 d^{2}$ & $1.00 \mathrm{i}$ & $2.67 \mathrm{~g}$ & $2.67 \mathrm{~g}$ & $3.00 \mathrm{fg}$ & $1.00 \mathrm{i}$ & $2.07 \mathrm{H}$ \\
\hline Mean & 2.64D & $4.28 \mathrm{~B}$ & 4.39B & $4.56 \mathrm{~A}$ & $4.14 \mathrm{C}$ & \\
\hline
\end{tabular}

The values within a column with different letters are significantly different at $\mathrm{P} \leq 0.001 \%$ according to the LSD multiple range test. 1: Cold storage period, 2: Cold storage period + shelf life 


\section{Total Chlorophyll}

Fig. 2 shows the effects of packages, storage duration and their interaction during two seasons on total chlorophyll. The lowest total chlorophyll was recorded in dill packed in non-perforated polypropylene package, while the highest content was recorded in perforated polypropylene package with 24 microholes. The results cleared that total chlorophyll significantly declined with elongation of cold storage and period of shelf life. Also, total chlorophyll was higher at each cold storage period at $0^{\circ} \mathrm{C}$ and duration of shelf life at $10^{\circ} \mathrm{C}$. Concerning the effect of interaction between packages and storage periods, the results indicated that total chlorophyll reduced sharply in non-perforated polypropylene packages with the cold storage at $0^{\circ} \mathrm{C}$ and period of shelf life at $10^{\circ} \mathrm{C}$. At the end of storage period, the perforated polypropylene package with 24 microholes had the highest total chlorophyll.

\section{Ascorbic Acid Content}

Table 4 results show the effects of packages, storage duration and their interaction $(\mathrm{p}<0.005)$ on ascorbic acid content in dill during two seasons. The non-perforated polypropylene package had the lowest ascorbic acid content during the two seasons, while no significant difference was detected between the perforated polypropylene package with 16 and 24 microholes in the first season. However, the perforated polypropylene package with 24 microholes had the highest value in the second season. Ascorbic acid decreased significantly during storage periods. With respect to the interaction, a severely decrease in ascorbic acid was detected in dill packed in the non-perforated polypropylene package during storage periods. After 28 days at $0^{\circ} \mathrm{C}$ and 4 days as shelf life at $10^{\circ} \mathrm{C}$, no significant differences were noticed between the perforated polypropylene packages with 8, 16, 24 and 32 microholes in the both seasons.

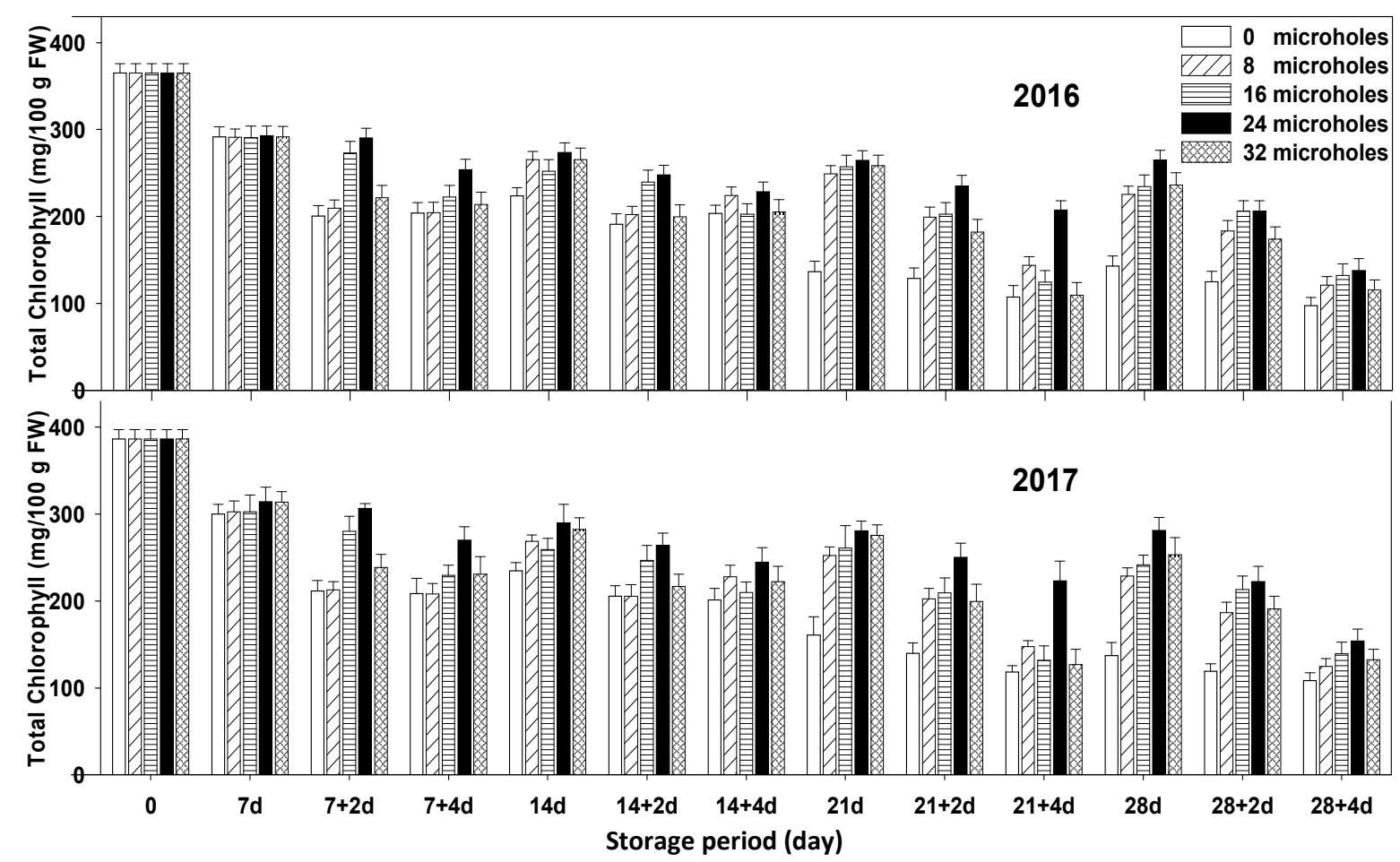

Fig. 2. Effect of microperforated polypropylene packages on total chlorophyll of dill during 2016 and 2017 seasons 
Zagazig J. Agric. Res., Vol. 46 No. (2) 2019

Table 4. Effect of microperforated polypropylene packages on ascorbic acid of dill during 2016 and 2017 seasons

\begin{tabular}{|c|c|c|c|c|c|c|}
\hline \multirow[t]{2}{*}{ Treatment } & \multicolumn{5}{|c|}{ Package } & \multirow[t]{2}{*}{ Mean } \\
\hline & Non-perforated & 8 holes & 16 holes & 24 holes & 32 holes & \\
\hline \multicolumn{7}{|c|}{2016 season } \\
\hline 0 time & 85.26 & 85.26 & 85.26 & 85.26 & 85.26 & \\
\hline $7 d^{1}$ & $63.09 \mathrm{~d}-\mathrm{g}$ & $74.41 \mathrm{a}$ & $74.54 \mathrm{a}$ & 73.68ab & $63.16 \mathrm{~d}-\mathrm{g}$ & $69.78 \mathrm{~A}$ \\
\hline $7+2 d^{2}$ & $52.56 \mathrm{i}-\mathrm{n}$ & $65.26 \mathrm{~b}-\mathrm{e}$ & 73.69ab & $63.09 \mathrm{~d}-\mathrm{g}$ & $57.89 \mathrm{~d}-\mathrm{k}$ & $62.50 \mathrm{~B}$ \\
\hline $7+4 d^{2}$ & $39.93 q-s$ & $58.88 \mathrm{~d}-\mathrm{k}$ & $50.53 \mathrm{k}-\mathrm{n}$ & $62.08 \mathrm{~d}-\mathrm{h}$ & $58.92 \mathrm{~d}-\mathrm{k}$ & $54.07 \mathrm{C}$ \\
\hline $14 d^{1}$ & $52.11 \mathrm{j}-\mathrm{n}$ & $60.53 d-j$ & $65.80 \mathrm{~b}-\mathrm{d}$ & $72.65 a-c$ & $64.67 c-f$ & $63.15 \mathrm{~B}$ \\
\hline $14+2 d^{2}$ & $41.55 \mathrm{o}-\mathrm{r}$ & $52.16 \mathrm{j}-\mathrm{n}$ & $58.75 \mathrm{~d}-\mathrm{k}$ & $60.63 \mathrm{~d}-\mathrm{i}$ & $52.64 \mathrm{i}-\mathrm{n}$ & $53.14 \mathrm{C}$ \\
\hline $14+4 d^{2}$ & $39.32 \mathrm{rs}$ & $56.84 \mathrm{e}-\mathrm{m}$ & $56.00 \mathrm{~g}-\mathrm{n}$ & $57.82 \mathrm{~d}-1$ & $57.86 \mathrm{~d}-\mathrm{k}$ & $53.57 \mathrm{C}$ \\
\hline $21 d^{1}$ & $52.64 \mathrm{i}-\mathrm{n}$ & $54.89 \mathrm{~g}-\mathrm{n}$ & $55.76 \mathrm{~g}-\mathrm{n}$ & $56.36 f-n$ & $52.37 \mathrm{i}-\mathrm{n}$ & $54.40 \mathrm{C}$ \\
\hline $21+2 d^{2}$ & $37.34 \mathrm{rs}$ & $52.86 \mathrm{i}-\mathrm{n}$ & $52.63 \mathrm{i}-\mathrm{n}$ & $54.64 h-n$ & $55.76 \mathrm{~g}-\mathrm{n}$ & $50.65 \mathrm{C}-\mathrm{E}$ \\
\hline $21+4 d^{2}$ & $40.63 p-r$ & 49.411-o & $53.90 \mathrm{~h}-\mathrm{n}$ & $57.86 \mathrm{~d}-\mathrm{k}$ & $53.57 \mathrm{i}-\mathrm{n}$ & $51.07 \mathrm{CD}$ \\
\hline $28 d^{1}$ & $48.42 \mathrm{~m}-\mathrm{p}$ & $52.60 \mathrm{i}-\mathrm{n}$ & 52.63i-n & $57.82 \mathrm{~d}-1$ & $52.64 \mathrm{i}-\mathrm{n}$ & $52.82 \mathrm{C}$ \\
\hline $28+2 d^{2}$ & $32.12 \mathrm{~s}$ & $52.79 \mathrm{i}-\mathrm{n}$ & 52.56i-n & $52.74 \mathrm{i}-\mathrm{n}$ & $52.56 \mathrm{i}-\mathrm{n}$ & $48.55 \mathrm{DE}$ \\
\hline $28+4 d^{2}$ & $33.64 \mathrm{rs}$ & $48.32 n-q$ & $52.04 \mathrm{k}-\mathrm{n}$ & $52.63 \mathrm{i}-\mathrm{n}$ & $49.01 \mathrm{~m}-\mathrm{p}$ & $47.13 \mathrm{E}$ \\
\hline Mean & $44.45 C$ & 56.58B & 58.24AB & $60.17 A$ & 55.92B & \\
\hline \multicolumn{7}{|c|}{2017 season } \\
\hline 0 time & 87.37 & 87.37 & 87.37 & 87.37 & 87.37 & \\
\hline $7 d^{1}$ & $65.34 d-g$ & $78.12 \mathrm{a}$ & $78.52 \mathrm{a}$ & $78.41 \mathrm{a}$ & $66.58 \mathrm{~d}-\mathrm{f}$ & 73.39A \\
\hline $7+2 d^{2}$ & $54.72 \mathrm{j}-\mathrm{n}$ & $68.67 \mathrm{~cd}$ & 77.37ab & $67.52 \mathrm{de}$ & $61.01 \mathrm{~d}-\mathrm{j}$ & $65.86 \mathrm{~B}$ \\
\hline $7+4 d^{2}$ & $42.07 \mathrm{pq}$ & $62.14 \mathrm{~d}-\mathrm{j}$ & $54.06 \mathrm{j}-\mathrm{n}$ & $66.36 \mathrm{~d}-\mathrm{f}$ & $61.89 \mathrm{~d}-\mathrm{j}$ & $57.31 \mathrm{C}$ \\
\hline $14 d^{1}$ & $53.74 \mathrm{j}-\mathrm{n}$ & $63.83 \mathrm{~d}-\mathrm{i}$ & $69.37 b-d$ & $76.97 a-c$ & $67.68 \mathrm{de}$ & $66.32 \mathrm{~B}$ \\
\hline $14+2 d^{2}$ & $43.07 \mathrm{o}-\mathrm{q}$ & $55.21 \mathrm{j}-\mathrm{n}$ & $62.07 \mathrm{~d}-\mathrm{j}$ & $64.70 \mathrm{~d}-\mathrm{h}$ & $55.40 \mathrm{i}-\mathrm{n}$ & $56.09 \mathrm{CD}$ \\
\hline $14+4 d^{2}$ & $39.71 \mathrm{qr}$ & $54.92 \mathrm{j}-\mathrm{n}$ & $59.24 \mathrm{e}-\mathrm{m}$ & $61.81 \mathrm{~d}-\mathrm{j}$ & $54.54 \mathrm{j}-\mathrm{n}$ & $54.04 \mathrm{C}-\mathrm{E}$ \\
\hline $21 d^{1}$ & $54.55 \mathrm{j}-\mathrm{n}$ & $57.87 \mathrm{~g}-\mathrm{n}$ & $59.01 \mathrm{f}-\mathrm{m}$ & $61.03 \mathrm{~d}-\mathrm{j}$ & $55.06 \mathrm{j}-\mathrm{n}$ & $57.50 \mathrm{C}$ \\
\hline $21+2 d^{2}$ & $38.81 \mathrm{qr}$ & 55.69i-n & 55.73i-n & $60.15 \mathrm{e}-\mathrm{k}$ & $54.30 \mathrm{j}-\mathrm{n}$ & $52.94 \mathrm{DE}$ \\
\hline $21+4 d^{2}$ & $38.96 \mathrm{qr}$ & $52.15 \mathrm{k}-\mathrm{n}$ & $53.91 \mathrm{j}-\mathrm{n}$ & $59.29 \mathrm{e}-1$ & $52.02 \mathrm{k}-\mathrm{n}$ & $51.27 \mathrm{EF}$ \\
\hline $28 d^{1}$ & $49.88 n-p$ & $55.46 \mathrm{i}-\mathrm{n}$ & 55.76i-n & $61.70 \mathrm{~d}-\mathrm{j}$ & $55.21 \mathrm{j}-\mathrm{n}$ & $55.60 \mathrm{CD}$ \\
\hline $28+2 d^{2}$ & $33.26 \mathrm{r}$ & 55.50i-n & $55.54 \mathrm{i}-\mathrm{n}$ & $56.47 \mathrm{~h}-\mathrm{n}$ & $54.98 \mathrm{j}-\mathrm{n}$ & $51.15 \mathrm{EF}$ \\
\hline $28+4 d^{2}$ & $32.75 \mathrm{r}$ & $50.80 \mathrm{~m}-\mathrm{o}$ & $54.79 \mathrm{j}-\mathrm{n}$ & 56.13i-n & 51.201-o & $49.13 \mathrm{~F}$ \\
\hline Mean & 45.57D & $59.20 \mathrm{BC}$ & $61.28 B$ & $64.21 \mathrm{~A}$ & $57.49 \mathrm{C}$ & \\
\hline
\end{tabular}

The values within a column with different letters are significantly different at $\mathrm{P} \leq 0.001 \%$ according to the LSD multiple range test. $\quad 1$ : Cold storage period, 2 : Cold storage period + shelf life. 


\section{Soluble Solids Content (SSC)}

The results in Table 5 show the effect of packages, storage period and their interaction on SSC of dill. Non-perforated polypropylene package had the lowest SSC compared with the other packages. No significant difference was observed between the perforated polypropylene package with 24 microholes and the perforated polypropylene package with 32 microholes. Also, no significant difference was noticed between the perforated polypropylene package with 8 microholes and the perforated polypropylene package with 16 microholes. However, the perforated polypropylene package with 32 microholes had the highest SSC. No clearly trend was observed in SSC during the duration of storage. However, the highest SSC was detected after period of cold storage for 21 days in both seasons. In regard to interaction, after 28 days at $0^{\circ} \mathrm{C}$ and 4 days at $10^{\circ} \mathrm{C}$, nonperforated polypropylene package had the lowest SSC followed by perforated polypropylene package with 8 microholes while no significant differences were detected between the perforated polypropylene packages with 16,24 and 32 microholes.

\section{Total Sugars}

Fig. 3 illustrates the effects of packages, storage periods and their interaction $(p<0.001)$ on dill total sugar contents after 28 days of cold storage at $0^{\circ} \mathrm{C}$ and 4 days of shelf life at $10^{\circ} \mathrm{C}$ during two seasons. The perforated polypropylene package with 24 microholes maintained the highest total sugars compared with the other packages. Total sugars decreased significantly during storage duration. Concerning the interaction, a sharp decrease in total sugars was detected in dill stored in non-perforated polypropylene package during the storage periods. At the end of storage, dill packed in the perforated polypropylene package with 24 microholes had the highest total sugars followed by the perforated polypropylene package with 32 microholes, while the lowest total sugars were recorded in dill packed in non-perforated polypropylene package.

\section{Total Phenolic}

The effect of packages, duration of storage and their interaction during both seasons was illustrated in Fig. 4. The results showed that the highest total phenolic was recorded in dill packed in the perforated polypropylene package with 24 microholes while the lowest was detected in non-perforated polypropylene package. Total phenolic reduced gradually with prolongation of storage duration at $0^{\circ} \mathrm{C}$ and period of shelf life at $10^{\circ} \mathrm{C}$. At the end of storage, a reduction in total phenolic was measured in all packages, but this reduction was higher in dill packed in nonperforated polypropylene package.

\section{DISCUSSION}

Dill is one of leafy vegetables which lose water at a higher rate than tubers and bulbs vegetables or soft fruit because are extremely vulnerable (Kays and Paull, 2004). The quality of most vegetables and fruit decreased very fast with small losses of moisture. In general, a loss of 3.00 to $10.00 \%$ may render a lot of horticultural crops unacceptable (Robinson et al., 1975). This study takes this into account, the weight loss of dill significantly reduced by perforated polypropylene packages. The weight loss ranged from 0.00 to $0.85 \%$ and 0.00 to $0.76 \%$ in both seasons, respectively. The highest weight loss (\%) in the perforated polypropylene packages with 32 microholes may attribute to the effect of higher microholes number on water loss. The effects of perforated polypropylene packages with 8,16 and 24 microholes on decreasing dill weight loss (\%) may attribute to reduce the transpiration rate by increasing water vapor pressure around the product and relative humidity inside the packages (Elwan et al., 2015). Also, the reduction in weight loss (\%) of dill packed in perforated polypropylene packages with 8,16 and 24 microholes could be due to less oxygen, which reduced the respiration rate, thereby lowering the loss of moisture by transpiration (Nath et al., 2011). This has been detected as a general effect of modified atmosphere packaging (MAP) on dill (Sakaldas et al., 2010), snap bean (Kinyuru et al., 2011), green bean (El-Bassiouny, 2003) and broccoli (Fernandez-Leon et al., 2013a).

The worst dill appearance was observed in non-perforated polypropylene package. This result may be due to the higher respiration rate and was not caused by weight loss because the weight loss in this package was low. This result is in agreement with the result of El-Bassiouny (2003) on snap beans, Lucera et al. (2011) on 
Zagazig J. Agric. Res., Vol. 46 No. (2) 2019

Table 5. Effect of microperforated polypropylene packages on SSC of dill during 2016 and 2017 seasons

\begin{tabular}{|c|c|c|c|c|c|c|}
\hline \multirow{2}{*}{ Treatment } & \multicolumn{5}{|c|}{ Package } & \multirow[t]{2}{*}{ Mean } \\
\hline & Non-perforated & 8 holes & 16 holes & 24 holes & 32 holes & \\
\hline \multicolumn{7}{|c|}{2016 season } \\
\hline 0 time & 4.00 & 4.00 & 4.00 & 4.00 & 4.00 & \\
\hline $7 d^{1}$ & 3.001 & $4.00 \mathrm{~d}-\mathrm{g}$ & $4.00 \mathrm{~d}-\mathrm{g}$ & $4.50 \mathrm{a}-\mathrm{c}$ & $3.80 \mathrm{e}-\mathrm{h}$ & 3.86B-E \\
\hline $7+2 d^{2}$ & $3.50 \mathrm{~h}-\mathrm{k}$ & 3.001 & $4.53 \mathrm{ab}$ & $4.00 \mathrm{~d}-\mathrm{g}$ & $4.00 \mathrm{~d}-\mathrm{g}$ & 3.81C-E \\
\hline $7+4 d^{2}$ & $3.53 \mathrm{~h}-\mathrm{j}$ & $3.80 \mathrm{e}-\mathrm{h}$ & $3.70 \mathrm{~g}-\mathrm{i}$ & $4.00 \mathrm{~d}-\mathrm{g}$ & $4.00 \mathrm{~d}-\mathrm{g}$ & 3.81C-E \\
\hline $14 d^{1}$ & $3.83 \mathrm{e}-\mathrm{h}$ & $4.00 \mathrm{~d}-\mathrm{g}$ & $3.17 \mathrm{j}-1$ & $4.13 c-f$ & $4.500 \mathrm{a}-\mathrm{c}$ & $3.93 \mathrm{~A}-\mathrm{D}$ \\
\hline $14+2 d^{2}$ & $3.67 \mathrm{~g}-\mathrm{i}$ & $4.00 \mathrm{~d}-\mathrm{g}$ & $4.00 \mathrm{~d}-\mathrm{g}$ & $4.00 \mathrm{~d}-\mathrm{g}$ & $4.00 \mathrm{~d}-\mathrm{g}$ & $3.93 \mathrm{~A}-\mathrm{C}$ \\
\hline $14+4 d^{2}$ & $3.13 \mathrm{kl}$ & $3.80 \mathrm{e}-\mathrm{h}$ & $3.77 \mathrm{f}-\mathrm{i}$ & $3.87 \mathrm{e}-\mathrm{h}$ & $4.00 \mathrm{~d}-\mathrm{g}$ & $3.71 \mathrm{E}$ \\
\hline $21 d^{1}$ & $3.83 \mathrm{e}-\mathrm{h}$ & $4.00 \mathrm{~d}-\mathrm{g}$ & $4.00 \mathrm{~d}-\mathrm{g}$ & $4.00 \mathrm{~d}-\mathrm{g}$ & $4.50 \mathrm{a}-\mathrm{c}$ & $4.07 \mathrm{~A}$ \\
\hline $21+2 d^{2}$ & 3.001 & $3.40 \mathrm{i}-\mathrm{k}$ & 3.001 & $4.00 \mathrm{~d}-\mathrm{g}$ & $4.00 \mathrm{~d}-\mathrm{g}$ & $3.48 \mathrm{~F}$ \\
\hline $21+4 d^{2}$ & 3.001 & $4.00 \mathrm{~d}-\mathrm{g}$ & $3.80 \mathrm{e}-\mathrm{h}$ & $4.00 \mathrm{~d}-\mathrm{g}$ & $4.50 \mathrm{a}-\mathrm{c}$ & 3.86B-E \\
\hline $28 d^{1}$ & $3.17 \mathrm{j}-1$ & $4.00 \mathrm{~d}-\mathrm{g}$ & $4.00 \mathrm{~d}-\mathrm{g}$ & $4.17 \mathrm{~b}-\mathrm{e}$ & $4.83 \mathrm{a}$ & $4.03 \mathrm{AB}$ \\
\hline $28+2 d^{2}$ & 2.931 & $3.83 \mathrm{e}-\mathrm{h}$ & $4.00 \mathrm{~d}-\mathrm{g}$ & $4.00 \mathrm{~d}-\mathrm{g}$ & $4.00 \mathrm{~d}-\mathrm{g}$ & $3.75 \mathrm{DE}$ \\
\hline $28+4 d^{2}$ & 2.871 & $3.67 \mathrm{~g}-\mathrm{i}$ & $4.33 \mathrm{~b}-\mathrm{d}$ & $4.00 \mathrm{~d}-\mathrm{g}$ & $4.00 \mathrm{~d}-\mathrm{g}$ & $3.77 \mathrm{C}-\mathrm{E}$ \\
\hline Mean & $3.29 \mathrm{C}$ & 3.79B & $3.86 \mathrm{~B}$ & $4.06 \mathrm{~A}$ & $4.18 \mathrm{~A}$ & \\
\hline \multicolumn{7}{|c|}{2017 season } \\
\hline 0 time & 4.10 & 4.10 & 4.10 & 4.10 & 4.10 & \\
\hline $7 d^{1}$ & $3.00 \mathrm{no}$ & $4.00 \mathrm{c}-\mathrm{h}$ & $4.00 \mathrm{c}-\mathrm{h}$ & $4.30 \mathrm{bc}$ & $4.03 \mathrm{c}-\mathrm{g}$ & $3.87 \mathrm{a}-\mathrm{d}$ \\
\hline $7+2 d^{2}$ & $3.33 \mathrm{k}-\mathrm{o}$ & $3.201-\mathrm{o}$ & $4.27 \mathrm{~b}-\mathrm{d}$ & $4.00 \mathrm{c}-\mathrm{h}$ & $4.00 \mathrm{c}-\mathrm{h}$ & $3.76 \mathrm{CD}$ \\
\hline $7+4 d^{2}$ & $3.40 \mathrm{j}-\mathrm{n}$ & 3.13 no & $3.93 c-i$ & $4.20 \mathrm{~b}-\mathrm{e}$ & $3.87 \mathrm{~d}-\mathrm{i}$ & 3.71DE \\
\hline $14 d^{1}$ & $3.70 \mathrm{~g}-\mathrm{k}$ & $4.00 \mathrm{c}-\mathrm{h}$ & $3.17 \mathrm{~m}-\mathrm{o}$ & $4.20 \mathrm{~b}-\mathrm{e}$ & $4.50 \mathrm{ab}$ & $3.91 \mathrm{~A}-\mathrm{C}$ \\
\hline $14+2 d^{2}$ & $3.60 \mathrm{~h}-1$ & $3.87 \mathrm{~d}-\mathrm{i}$ & $3.87 \mathrm{~d}-\mathrm{i}$ & $4.20 \mathrm{~b}-\mathrm{e}$ & $4.13 b-f$ & $3.93 \mathrm{~A}-\mathrm{C}$ \\
\hline $14+4 d^{2}$ & 3.13 no & $3.80 \mathrm{e}-\mathrm{j}$ & $3.60 \mathrm{~h}-1$ & $4.00 \mathrm{c}-\mathrm{h}$ & $4.00 c-h$ & $3.71 \mathrm{DE}$ \\
\hline $21 d^{1}$ & $3.77 \mathrm{f}-\mathrm{j}$ & $4.00 \mathrm{c}-\mathrm{h}$ & $4.00 \mathrm{c}-\mathrm{h}$ & $4.10 \mathrm{~b}-\mathrm{g}$ & $4.33 b c$ & $4.04 \mathrm{~A}$ \\
\hline $21+2 d^{2}$ & $3.00 \mathrm{no}$ & $3.40 \mathrm{j}-\mathrm{n}$ & 3.201-o & $4.00 \mathrm{c}-\mathrm{h}$ & $4.17 b-f$ & $3.55 \mathrm{E}$ \\
\hline $21+4 d^{2}$ & $3.00 \mathrm{no}$ & $4.00 \mathrm{c}-\mathrm{h}$ & $3.70 \mathrm{~g}-\mathrm{k}$ & $4.17 \mathrm{~b}-\mathrm{f}$ & $4.50 \mathrm{ab}$ & $3.87 \mathrm{~A}-\mathrm{D}$ \\
\hline $28 d^{1}$ & 2.930 & $4.00 \mathrm{c}-\mathrm{h}$ & $4.00 \mathrm{c}-\mathrm{h}$ & $4.17 \mathrm{~b}-\mathrm{f}$ & $4.77 \mathrm{a}$ & $3.97 \mathrm{AB}$ \\
\hline $28+2 d^{2}$ & $3.00 \mathrm{no}$ & $3.83 \mathrm{e}-\mathrm{i}$ & $4.07 \mathrm{c}-\mathrm{g}$ & $4.00 \mathrm{c}-\mathrm{h}$ & $4.20 \mathrm{~b}-\mathrm{e}$ & 3.82B-D \\
\hline $28+4 d^{2}$ & 2.930 & $3.57 \mathrm{i}-\mathrm{m}$ & $4.00 \mathrm{c}-\mathrm{h}$ & $4.10 \mathrm{~b}-\mathrm{g}$ & $4.30 \mathrm{bc}$ & $3.78 \mathrm{CD}$ \\
\hline Mean & $3.23 \mathrm{C}$ & 3.73B & 3.81B & $4.12 \mathrm{~A}$ & $4.23 \mathrm{~A}$ & \\
\hline
\end{tabular}

The values within a column with different letters are significantly different at $\mathrm{P} \leq 0.001 \%$ according to the LSD multiple range test. 1: Cold storage period, 2: Cold storage period + shelf life. 

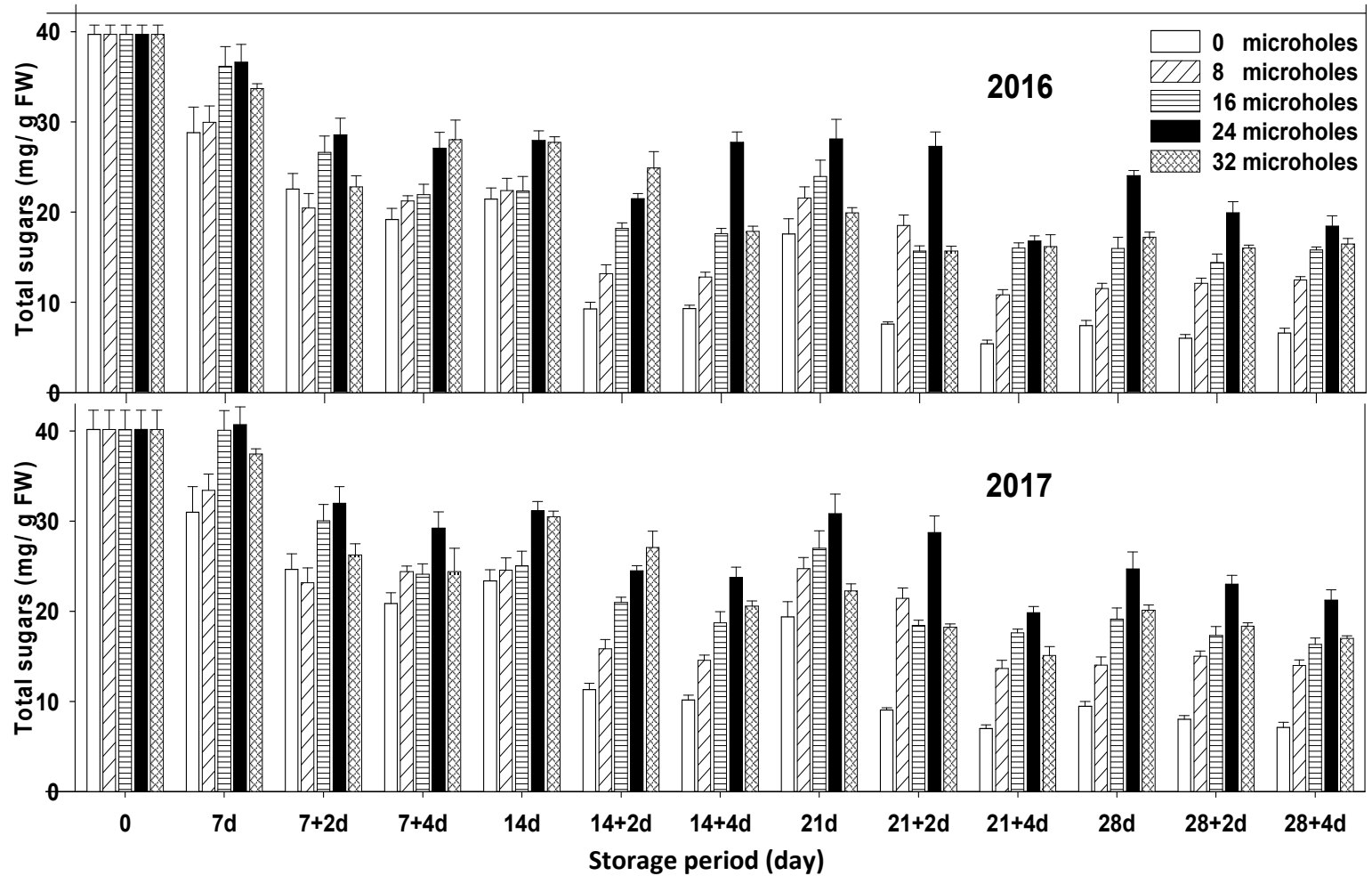

Fig. 3. Effect of microperforated polypropylene packages on total sugars of dill during 2016 and 2017 seasons



Fig. 4. Effect of microperforated polypropylene packages on total phenolic of dill during 2016 and 2017 seasons 
fresh-cut green beans and Elwan et al. (2015) on snap peas. The best appearance was observed in the perforated polypropylene package with 24 microholes (24 hr.). The good visual quality has been recorded in modified atmosphere packaging (Sakaldas et al., 2010). This result suggests that the perforated polypropylene package with 24 microholes (24 hr.) maintained the quality of dill by gas composition inside the package.

The highest off odor was detected in nonperforated polypropylene package. The increase in off odor into this package may attribute to the decrease in $\mathrm{O}_{2}$ and the increase in $\mathrm{CO}_{2}$ concentrations by increasing the respiration rate which triggers anaerobic conditions, thus the anaerobic respiration quickly destroys the quality of produce by tissue breakdown, accumulation of acetaldehyde and ethanol and development of off-odor (Elwan et al., 2015). The tissue will start anaerobic respiration when the oxygen partial pressure in MAP reduces below the threshold limit of fermentation which produces off odors (Soliva-Fortuny et al., 2004). Off odor was detected in sealed polyethylene package during storage period of green beans (El-Bassiouny, 2003). The lowest off odor was recorded in the perforated polypropylene package with 24 microholes. This indicates that this package provides a good gas composition by its appropriate permeability, depending on the number and diameter of microholes.

The flavor of dill is an important quality attributes, packed dill in the perforated polypropylene package with 24 microholes had the best taste while those packed in nonperforated polypropylene package had the worst taste. Similarly, Elwan et al. (2015) stated that the better taste of sugar snap peas was obtained with micro-perforated polypropylene with 12 microholes compared to non-perforated polypropylene package which resulted in lost the sugar snap peas taste at the end of storage time. These results designate that the perforated polypropylene package with 24 microholes preserved the quality of dill.

Chlorophyll is the importance factor to judge the quality of leafy vegetables. Modified atmosphere packaging delayed the decline of dill chlorophyll content (Sakaldas et al., 2010).
In this study, dill packed in the perforated polypropylene package with 24 microholes had the highest total chlorophyll content. This result is in harmony with the result of Nath et al. (2011) on broccoli and Elwan et al. (2015) on sugar snap peas. The quicker loss in total chlorophyll was observed in dill packed in nonperforated polypropylene package which had the lowest total chlorophyll during storage and duration of shelf life. The effect of nonperforated polypropylene package may be due to the accumulation of $\mathrm{CO}_{2}$ via increasing the respiration rate, which results in the chlorophyll degradation. Also, the reduction in total chlorophyll is considered typical during the senescence process (Gross, 1991).

The reduction of ascorbic acid during storage period may attribute to the oxidation by enzymes such as polyphenol oxidase and ascorbic acid oxidase which reduce the ascorbic acid of vegetables (Mapson (1970), and convert it into dehydroascorbic acid (Albuquerque et al., 2005). The highest ascorbic acid content in packed dill in perforated polypropylene package with 24 microholes may be attributed to the atmospheric oxygen limitation available for oxidation. The same result was informed by Kinyuru et al. (2011) on snap beans, Fernandez-Leon et al. (2013a,b) on broccoli and Elwan et al. (2015) on sugar snap peas.

The lowest SSC was observed in packed dill in non-perforated polypropylene package. This reduction in SSC may be due to the effect of more carbon dioxide and less oxygen in nonperforated package, generating a high rate of respiration (Del-Valle et al., 2009), which resulting in sugars consumption via glycolysis and hydrolysis reactions (Bodelon et al., 2010). On the other hand, perforated polypropylene packages with 24 and 32 microholes were more effective in keeping SSC by reducing the respiration rate of dill.

MAP retards the loss of sugars by reducing respiration rate (Elwan et al., 2015). In this study, the highest total sugars were recorded in dill stored in perforated polypropylene package with 24 microholes. Also, the perforated polypropylene packages decreased $\mathrm{CO}_{2}$ level and postponed carbohydrate metabolism (Aday et al., 2011). Similarly Escalona et al. (2004) on 
fennel and Gomez and Artes (2005) with celery. The sharp decrease in total sugars content of stored dill in non- perforated polypropylene package may be due to the consumption of sugars by the higher respiration rate.

Modified atmosphere packaging prevented the phenolic compounds degradation (Sakaldas et al., 2010). The perforated polypropylene package with 24 microholes had the highest total phenolic of dill. This result could be attributed to low oxygen and high carbon dioxide which caused a decrease in activities of polyphenol oxidase and peroxidase, thus preventing the phenolic degradation (Saxena et al., 2009).

\section{Conclusion}

The perforated polypropylene package with 24 microholes greatly extended the storage period of dill at $0^{\circ} \mathrm{C}$ for 28 days and shelf life at $10^{\circ} \mathrm{C}$ for 4 days with good appearance and taste and had the highest value for each of total chlorophyll, ascorbic acid, sugars and phenolic and the lowest off-odor.

\section{REFERENCES}

Aday, M.S., C. Caner and F. Rahvalı (2011). Effect of oxygen and carbon dioxide absorbers on strawberry quality. Postharvest Biol. and Technol., 62: 179-187.

Albuquerque, B., F.C. Lidon and M.G. Barreiro. (2005). A case study of tendral winter melons (Cucumis melo L.) Post harvest senescence. General and Appl. Plant Physiol., 31: 157-169.

AOAC (1996). Official Methods of Analysis, Association of Official Analytical Chemists, Washington DC.

Bodelon, O.G., M. Blanch, M.T. SanchezBallesta, M.I. Escribano and C. Merodio (2010). The effects of high $\mathrm{CO}_{2}$ levels on anthocyanin composition, antioxidant activity and soluble sugar content of strawberries stored at low non-freezing temperature. Food Chem., 122: 673-678.

Church, I. and A. Parsons (1995). Modified atmosphere packaging technology: Review. J. Sci. and Food and Agric., 67:143-152.
Del-Valle, V., P. Hernandez-Munoz, R. Catala and R. Gavara (2009). Optimization of an equilibrium modified atmosphere packaging (EMAP) for minimally processed mandarin segments. J. Food Eng., 91: 474-481.

Dubois, M.K.A., J.K. Hamilton, P.A. Rebers and F. Smith (1956). Colorimetric method for determination of sugars and related substances, Anal. Chem., 28 (3): 350-356.

El-Bassiouny, R.E.I. (2003). Modified atmosphere polyethylene packages maintain the quality of snap bean pods during storage. J. Agric. Sci. Mansoura Univ., 28 (5): 3869-3888.

Elwan, M.W.M., I.N. Nasef, S.K. El-Seifi, Hassan, M.A. and R.E. Ibrahim (2015). 'Storability, shelf-life and quality assurance of sugar snap peas (cv. Super sugar snap) using modified atmosphere packaging', Postharvest Biol. Technol., 100: 205-211.

Escalona, V.H., E. Aguayo, P. Gomez and F. Artes (2004). Modified atmosphere packaging inhibits browning in fennel. Lebensmittel Wissenschaft und-Technol., 37: 115-121.

Fernandez-Leon, M.F., A.M. Fernandez-Leon, M. Lozano, M.C. Ayuso and D. GonzalezGomez (2013a). Altered commercial controlled atmosphere storage conditions for 'Parhenon' broccoli plants (Brassica oleracea L. var. italica). Influence on the outer quality parameters and on the health-promoting compounds. Food Sci. and Technol., 50: 665672.

Fernandez-Leon, M.F., A.M. Fernandez-Leon, M. Lozano, M.C. Ayuso and D. GonzalezGomez (2013b). Different postharvest strategies to preserve broccoli quality during storage and shelf life: Controlled atmosphere and 1-MCP. Food Chem., 138: 564-573.

Gomez, P.A. and F. Artes (2005). Improved keeping quality of minimally fresh processed celery sticks by modified atmosphere packaging. Lebensmittel Wissenschaft undTechnol., 38: 323-329.

Gross, J. (1991). Pigments in Vegetables. New York: Van Nostrand Reinhold.

Jiang, W., Q. Sheng, X. Zhou, M. Zhang and X. Liu (2002). Regulation of detached coriander 
leaf senescence by 1-methylcyclopropene and ethylene. Postharvest Biol. Tech-nol., 26: 339-345.

Kays, S.J. and R.E. Paull (2004). Stess in harvested products, p. 355-414. In: S.J. Kays and R.E. Paull (eds.). Postharvest biology. Exon Press, Athens, GA.

Kinyuru, J.N., K.P. Kahenya, M. Muchui and H. Mungai (2011). Influence of post-harvest handling on the quality of snap bean (Phaseolus vulgaris L.). J. Agric. and Food Technol., 1 (5): 43-46.

Koukounaras, A., A.S. Siomos and E. Sfakiotakis (2006). 1-Methylcyclopropene prevents ethylene induced yellowing of rocket leaves. Postharvest Biol. and Technol., 41: 109-111.

Koukounaras, A., G. Diamantidis and E. Sfakiotakis (2008). The effect of heat treatment on quality retention of fresh-cut peach. Postharvest Biol. and Technol., 48: 30-36.

Lers, A., W. Jiang, E. Lomaniec, N. Aharoni, (1998). Gibberellic acid and $\mathrm{CO}_{2}$ additive effect in retarding postharvest senescence of parsley. J. Food Sci., 63: 66-68.

Lichenthaler, H.K. and W.R. Wellburn (1983). Determination of total carotenoids and chlorophylls $\mathrm{a}$ and $\mathrm{b}$ of leaf extracts in different solvents. Biochem. Soc. Trans., 11: 591-592.

Lucera, A., A. Conte and M.A. Del Nobile (2011). Shelf life of fresh-cut green beans as affected by packaging systems. Int. J. Food Sci. and Technol., 46 : 2351-2357.

Mapson, C.W. (1970). Vitamins in Fruits: Stability of L-ascorbic acid. In Biochemistry of Fruits and Their Products London: Academic Press, 376-387.

Mazumdar, B.C. and K. Majumder (2003). Methods on Physico-Chemical Analysis of Fruits. Daya Publishing House, Delhi110035, 162-163.
Nath, A., B. Bagchi, L.K. Misra and B. C. Deka (2011). Changes in post-harvest phytochemical qualities of broccoli florets during ambient and refrigerated storage. Food Chem., 127: 1510-1514.

Pearson, D. (1970). The Chemical Analysis of Foods ( $6^{\text {th }}$ Ed.), T.A Constable, London.

Robinson, J.E., K.M. Browne and W.G. Burton (1975). Storage characteristics of some vegetables and soft fruits. Ann. Appl. Biol., 81: 399-408.

Sakaldas, M., A.Ş. Aslım, C.O. Kuzucu and K. Kaynas (2010). The effects of modified atmosphere packaging and storage temperature on quality and biochemical properties of dill (Anethum graveolens) leaves. J. Food, Agric. and Environ., 8 (3,4): 21- 25 .

Sanz, C., A.G. Perez, R. Olias and J.M. Olias (1999). Quality of strawberries packaged with perforated polypropylene. J. Food Sci., 64 (4): 748-752.

Saxena, A., A.S. Bawa and P.S. Raju (2009). Phytochemical changes in fresh-cut jackfruit (Artocarpus heterophyllus L.) bulbs during modified atmosphere storage. Food Chem., 115 (4): $1443-1449$.

Soliva-Fortuny, R.C., N. Alos-Saiz, A. EspachsBarroso and O. Martin-Belloso (2004). Influence of maturity at processing on quality attributes of fresh-cut conference pears. J. Food Sci., 69 (7): 290-294.

Zagory, D. (1997). Advances in Modified Atmosphere Packaging (MAP) of Fresh Produce. Perishables Handling Newsletter, Univ. Calif. Davis CA, 90: 2- 4.

Zagory, D. (1998). An Update on Modified Atmosphere Packaging of Fresh Products. Pack. Int., 117. 
زيادة فترة التخزين والعرض والحفاظ على جودة الشبت باستخدام عبوات البولى بروبيلين المثقبة تثقيباً دقيقاً

سمير كامل الصيفي' - ـراوية البسيونى إبراهيم البسيوني '- إبراهيم ناصف ناصف ' ـ تسنيم حمادة حمادة'



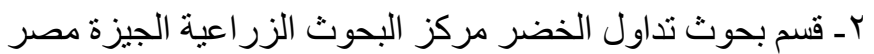

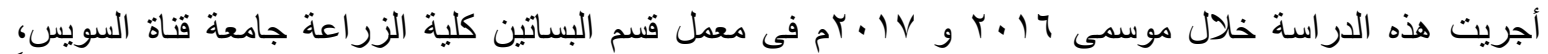





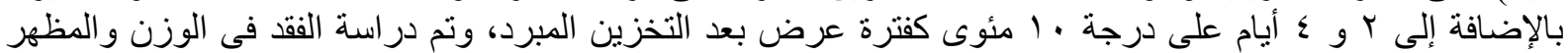

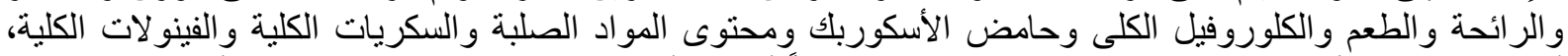

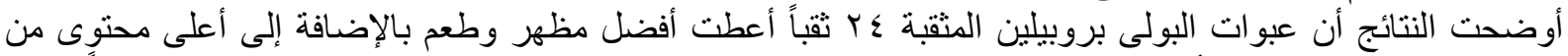

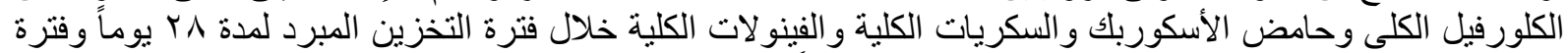



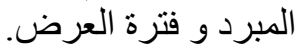

